Didáctica Geográfica n 19, 2018,pp. 103-125

ISSN: 0210-492-X

D.L: M-3736-2014

\title{
SE HACE GEOGRAFÍA AL ANDAR: LA SALIDA DE CAMPO ITINERANTE Y SENDERISTA
}

Miguel García Martín'; Arsenio Villar Lama²; Pablo Fraile Jurado; Noela Sánchez Carnero ${ }^{4}$ Joaquín Márquez Pérez ${ }^{5}$

Recibido: 23/11/2017

Aceptado: 01/07/2018

\section{RESUMEN:}

Las salidas de campo representan un método de enseñanza fuera del aula muy característico de la Geografía y cuentan con una larga trayectoria histórica. En este artículo presentamos la Salida Itinerante de Geografía, una experiencia docente transversal en la que profesores y alumnos universitarios recorren a pie durante varios días seguidos un espacio de carácter natural y rural. La caminata sirve para vertebrar el trabajo de campo, que se organiza por grupos, abordando las distintas claves territoriales y paisajísticas del ámbito. Asimismo, llevamos a cabo una evaluación de esta experiencia docente que servirá para mejorar futuras ediciones.

\section{Palabras Clave:}

Salida de campo, transversalidad, senderismo, aprendizaje cooperativo, espacios naturales protegidos.

${ }^{1}$ Departamento de Geografía Humana, Universidad de Sevilla. mgmartin@us.es. Facultad de Geografía e Historia, C/María de Padilla s/n, 41004 Sevilla

2 Departamento de Geografía Física y AGR, Universidad de Sevilla. arsenio@us.es

3 Departamento de Geografía Física y AGR, Universidad de Sevilla.pfraile@us.es

${ }^{4}$ Centro de Estudios de Sistemas Marinos (CESIMAR, Argentina).noelas@gmail.com

5 Departamento de Geografía Física y AGR, Universidad de Sevilla.jmarper@us.es 


\section{Abstract:}

The field trips represent a method of teaching outside the classroom, which is very characteristic in Geography and have a long history. In this paper we present the Salida Itinerante de Geografía, a cross-cutting teaching experience where university teachers and students walk for several days through natural and rural areas. The hike serves to vertebrate the field work, which is organized by groups and themes, addressing the different territorial keys of the area. Likewise, an evaluation of this teaching experience is carried out as a feedback to improve future editions.

\section{KEYWORDS:}

Field trip, transversality, cooperative learning, trekking, natural protected areas.

\section{RÉSUMÉ:}

Les sorties de terrain constituent une méthode d'enseignement en dehors de la salle de cours caractéristiques de la Géographie qui ont une longue trajectoire historique. Dans cet article, nous présentons la Salida Itinerante de Geografía, une expérience d'enseignement transversale dans laquelle les enseignants et les élèves du parcourent un espace à caractère naturel et rural. La randonnée sert à structurer le travail sur le terrain, organisé en groupes qui abordent les aspectsles plus remarquables du parcours du point de vue territorial. En outre, nous avons effectué une évaluation de cette expérience éducative qui serviront de feedback à appliquer dans les éditions à venir.

\section{Mots-Clés:}

Sortie de terrain, enseignement transversal, apprentissage coopératif, randonnée, espaces naturels protégés.

\section{INTRODUCCIÓN}

La salida de campo constituye un método didáctico fundamental en la enseñanza de la Geografía, así como en sus aplicaciones profesionales. Su importancia estriba en que proporciona a los estudiantes una experiencia práctica del mundo real a través de culturas y territorios (Fuller, Edmondson, France, Higgitt \& Ratinen, 2006) o, como señala Barraqué Nicolau (1978), vincula la escuela con la vida, convirtiendo la realidad en un medio de enseñanza.

Como método trasciende las competencias propias más clásicas de las ciencias geográficas, a saber, la observación, la descripción y el análisis, para servir de eje conductor a la interpretación y la lectura hermenéutica de la manifestación sensible del 
territorio: el paisaje (Delgado Bujalance y Ojeda Rivera, 2007). Pero la presencia in situ de docentes y alumnos también favorece la motivación como catalizador del proceso de aprendizaje y enseñanza, creando un compromiso ético y social por medio de una valoración activa y comprometida de los significados inherentes a todo paisaje (García de la Vega, 2004; Sousa Fernandes, García Monteagudo \& Souto González, 2016). En última instancia, desplazar la práctica docente al campo - al afueras - convierte a los alumnos en actores en cierta medida partícipes del tablero de juego que es el espacio geográfico (García Martín, 2014), por su parentesco con un ejercicio de investigación participante.

Así, bajo el amplio espectro de la práctica didáctica considerada fuera del aula, se distinguen diversas experiencias que se diferencian unas de otras en su tratamiento instrumental, en la escala espacial y temática de trabajo, en el grado de integración interdisciplinar e incluso en el distinto compromiso pedagógico y social. Es por ello que el abanico terminológico es amplio, distinguiendo (en ocasiones de forma confusa) entre la salida de campo, el itinerario didáctico, la excursión pedagógica, el trabajo de campo, el viaje o la excursión geográfica, etc.; si bien la primera de estas acepciones ha acabado imponiéndose como término y fórmula en los currículos educativos, especialmente en la enseñanza superior.

Estas experiencias, a las que Gómez Mendoza y Sanz Herráiz (2013) se refieren como excursión geográfica universitaria, poseen una base histórica muy sólida: sus precedentes se remontan a los viajes de los naturalistas en los siglos XVIII y XIX, el viaje geográfico de la Escuela Francesa (Vidal de la Blache, De Martonne) y su influencia sobre la Societat Catalana de Geografía (Pau Vila, Lluis Solé), ya en el siglo XX. En aquellas expediciones científicas, la perspectiva física y, especialmente, la geomorfológica eran claramente preponderantes. La difusión del viaje pedagógico al conjunto de la Geografía española está muy relacionada con las Reuniones de Estudios Geográficos. Manuel de Terán, en el marco de la Institución Libre de Enseñanza, y Martínez de Pisón serán dos personajes claves en el impulso del viaje geográfico: el primero, por la búsqueda del equilibrio entre el estudio físico y humano del territorio; y ambos, por la progresiva institucionalización de la actividad y su integración en los itinerarios curriculares universitarios. Aquellas innovaciones llegaron a los niveles educativos más básicos que, en la forma de paseos escolares, alternaban las funciones didácticas con un fomento del ejercicio como práctica higienista (Almuedo Palma, 2016).

Así pues, aquellas formas de enseñanza fuera del aula que, particularmente, incorporan largas caminatas a pie como hilo conductor de la actividad, pueden llegar a enriquecer significativamente este método didáctico. En estos casos, el itinerario se convierte en un medio de acceso a los paisajes y sus elementos configuradores (ya sea una formación geológica, un aprovechamiento agrícola o bien se trate de observar variaciones en la 
temperatura del aire); pero también es un fin en sí mismo, por cuanto que el camino y el caminar constituyen formas propias de conocimiento, y solo caminando - y dejando a menudo de hacerlo, es decir, parándose - se llega a aprehender ciertos fenómenos que explican el complejo orden espacial de los territorios. Precisamente porque la escala de las cosas percibidas cambia con la manera de moverse y la velocidad.

El caminar es hasta tal punto un fin en sí mismo que puede considerarse una práctica estética - como así la entendieron por ejemplo las vanguardias modernistas que practicaban la flânerie o los artistas del Land Art (Careri, 2002) - o también una práctica ética, por cuanto que humaniza a las personas, volviendo "más habitable este mundo ante unos valores que cada vez priorizan más lo que transmite el espíritu automovilístico y tecnológico (inmediatez, velocidad, superficialidad o ligereza)" (García Farrero, 2014, p. 78). Son muchos los filósofos, pensadores y literatos que a lo largo del tiempo han defendido la prácica caminante como catalizadora del pensamiento, el conocimiento o la inspiración, incluso como forma de liberación: Jean-Jaques Rousseau, Emmanuel Kant, Henry David Thoreau, Friedrich Nietzsche - quien desconfiaba de aquellos pensamientos que no surgieran al aire libre, andando, en movimiento-, Robert Louis Stevenson, Walter Benjamin... son solo algunos ejemplos (Gros, 2014; Solnit, 2015). En efecto, la psicología cognitiva ha puesto de manifiesto que el acto de caminar al aire libre puede favorecer la estimulación de ideas e incrementar la creatividad (Oppezzo y Schwartz, 2014).

Independientemente de lo anterior, caminar es de las actividades que mejor aprovechan las ventajas de la práctica docente fuera del aula. Así, la naturaleza transdisciplinar del conocimiento geográfico encuentra en las salidas de campo, a pie, un medio idóneo de aplicación didáctica de las distintas materias por cuanto que el itinerario atraviesa y conecta física pero también conceptualmente los distintos escenarios (ecosistemas, ambientes, paisajes, regiones) por los que se transita; en especial cuando estos itinerarios son de largo recorrido, incluso prolongándose por espacio de varios días. Es en este contexto pedagógico donde la visión holística del conocimiento geográfico adquiere toda su dimensión, pues se pueden percibir, e incluso padecer, los condicionantes físiconaturales y sus manifestaciones humanas resultantes a una escala de mucho detalle.

Actualmente parecen convivir en los viajes geográficos de formación académica dos tendencias complementarias: de una parte, las salidas de campo especializadas, ligadas directamente a materias específicas y, de otro, nuevas propuestas multi y transdisciplinares. En esta línea llaman nuestra atención aquellas actividades que se vienen desarrollando en los últimos años y conforman una referencia para nosotros, como por ejemplo, los itinerarios didácticos de la Sierra de Guadarrama (Crespo Castellanos, 2012) o los viajes pedagógicos por Andalucía desde la Universidad Pablo de Olavide, donde se incide en el papel del paisaje como hilo conductor del aprendizaje (Delgado Bujalance et al., 2013). 
La incorporación a la dimensión científica de las ya mencionadas dimensiones ética y estética - en definitiva, el paisaje - conforma una de las ideas clave de esta propuesta.

La revisión bibliográfica permite afirmar que este particular recurso didáctico es motivo de inspiración y reflexión para los docentes que, según Lara de González (2011), sintetizan en dos los pilares sobre los que debe asentarse: un excelso trabajo de planificación previa y el establecimiento de unos objetivos que favorezcan un aprendizaje significativo. En esta línea - la de motivar y significar para aprenderPérez de Sánchez y Rodríguez Pizzinato (2006) enfatizan la relevancia de las preguntas iniciales "problematizadoras", ya que motivan a la indagación documental y al contacto real con el ámbito (y el problema) durante la propia salida.

Así, parece evidente lo importante del viaje geográfico como método pedagógico, su dilatada trayectoria y la variedad de versiones y escuelas. En consecuencia, el objetivo de este trabajo es exponer las principales particularidades didácticas de la Salida de Campo Itinerante Senderista de Geografía (en lo sucesivo, Salida Itinerante), evaluar su utilidad y plantear perspectivas de mejora en el futuro. Se recurre para ello a la estrategia de mejora contínua basada en el ciclo PDCA (Plan, Do, Check, Act), por la cual, como fases posteriores a la planificación y a la ejecución de una actividad docente, se deben evalúar sus contenidos para proponer líneas de mejora que se apliquen en ocasiones posteriores. En consonancia, este artículo recoje el testigo de una comunicación presentada en el XI Congreso de Didáctica de la Geografía (Marquez et al., 2016), enriqueciéndose con algunas otras experiencias allí debatidas así como con la ampliación de la muestra de alumnos encuestados para la evaluación de esta actividad didáctica.

\section{DESCRIPCIÓN DE LA ACTIVIDAD}

\subsection{Definición, objetivos y contenidos}

La Salida Itinerante consiste en la realización de una salida de campo de varios días de duración, a pie y por un espacio de carácter natural y rural, donde profesores y alumnos de la titulación de Geografía, previamente organizados en grupos de trabajo, analizan las principales claves territoriales del ámbito recorrido. Los resultados se comparten mediante las exposiciones temáticas de los distintos grupos.

Se trata de una actividad de campo semi-dirigida. Siguiendo a Godoy y Sánchez (2007) - que diferencian entre dirigidas, semi-dirigidas y sin dirección-, en este tipo de actividad el papel del profesorado es importante en el establecimiento previo del recorrido, en la exposición de las claves iniciales del ámbito y en la acción tutorial, siendo el alumnado (individualmente o en equipo) el que protagoniza la mayor parte del proceso: indaga en las fuentes, sintetiza las ideas, plantea los objetivos y presenta los principales resultados. 
Desde el curso 2007-08 se han realizado 10 ediciones, una por cada curso (Tabla $\mathrm{n}^{\mathrm{o}} 1$ ). En total se han recorrido unos 650 kilómetros en diversos espacios naturales en España, mayoritariamente en Andalucía (Figura ${ }^{\circ}{ }^{2}$ ), llevándose a cabo un total de 40 jornadas de campo. En la actividad ha participado un total de 144 estudiantes, si bien una parte significativa (33) ha estado presente en varias ediciones. Los Departamentos de Geografía de la Universidad de Sevilla amparan institucionalmente la actividad, sufragando una parte importante de los gastos. El resto hasta completar el presupuesto es aportado por todos los participantes. Desde el punto de vista del itinerario curricular del alumno, este evento ha sido reconocido como "Asignatura de Libre Configuración" (2011-2012) y como "Actividad con Reconocimiento Académico" en la titulación de Grado (2014-2016), con 2 ECTS.

\begin{tabular}{|c|l|l|l|c|}
\hline Año & \multicolumn{1}{|c|}{ Localización } & \multicolumn{1}{c|}{ Ruta } & \multicolumn{1}{c|}{ Itinerario } & Km \\
\hline 2008 & Alpujarra Granadina & GR-7 & Trévelez-Lanjarón & 51 \\
\hline 2009 & Alpujarra Granadina & GR-7 & Laroles-Trévelez & 56 \\
\hline 2010 & Sierra de Segura & GR-147 & Santiago de la Espada-Segura S. & 70 \\
\hline 2011 & Serranía de Cuenca & GR-66 & Tragacete-Cuenca & 68 \\
\hline 2012 & Sierra de Segura & GR-7 & Venta Ticiano-Cotorríos & 70 \\
\hline 2013 & Sierra de Huelva & $\begin{array}{l}\text { PR A 004, } \\
\text { 038 y 039 }\end{array}$ & Aracena-Cortegana & 64 \\
\hline 2014 & Sierra de Grazalema & PR A 347 & Grazalema-Grazalema & 45 \\
\hline 2015 & Valle del Genal & $\begin{array}{l}\text { PR A 222, } \\
\text { 224, 235-240 }\end{array}$ & Parauta-Atajate & 66 \\
\hline 2016 & Sierra de las Nieves & GR 243 & El Burgo-Tolox & 82 \\
\hline 2017 & Sierra de Cazorla y el Pozo & GR 247 & Cazorla-Cazorla & 74 \\
\hline
\end{tabular}

TABLA No 1: Cuadro-Resumen de la Salida Itinerante de Geografía 2008-2017

El objetivo principal de la Salida es visualizar, consolidar y aprehender los contenidos integrados en los estudios universitarios de Geografía, aprovechando la inmediatez de la realidad estudiada. El hecho de colocar al alumnado en una posición en la que pueden confluir muchos aspectos distintos de una misma realidad (académicamente hablando, muchas asignaturas) les refuerza la idea de la Geografía como una disciplina "transversal". Entre los objetivos específicos de la Salida destacan:

a) Facilitar que muchos de los conocimientos que se imparten de forma teórica en la carrera puedan ser, si no aplicados, al menos visualizados y contextualizados. 


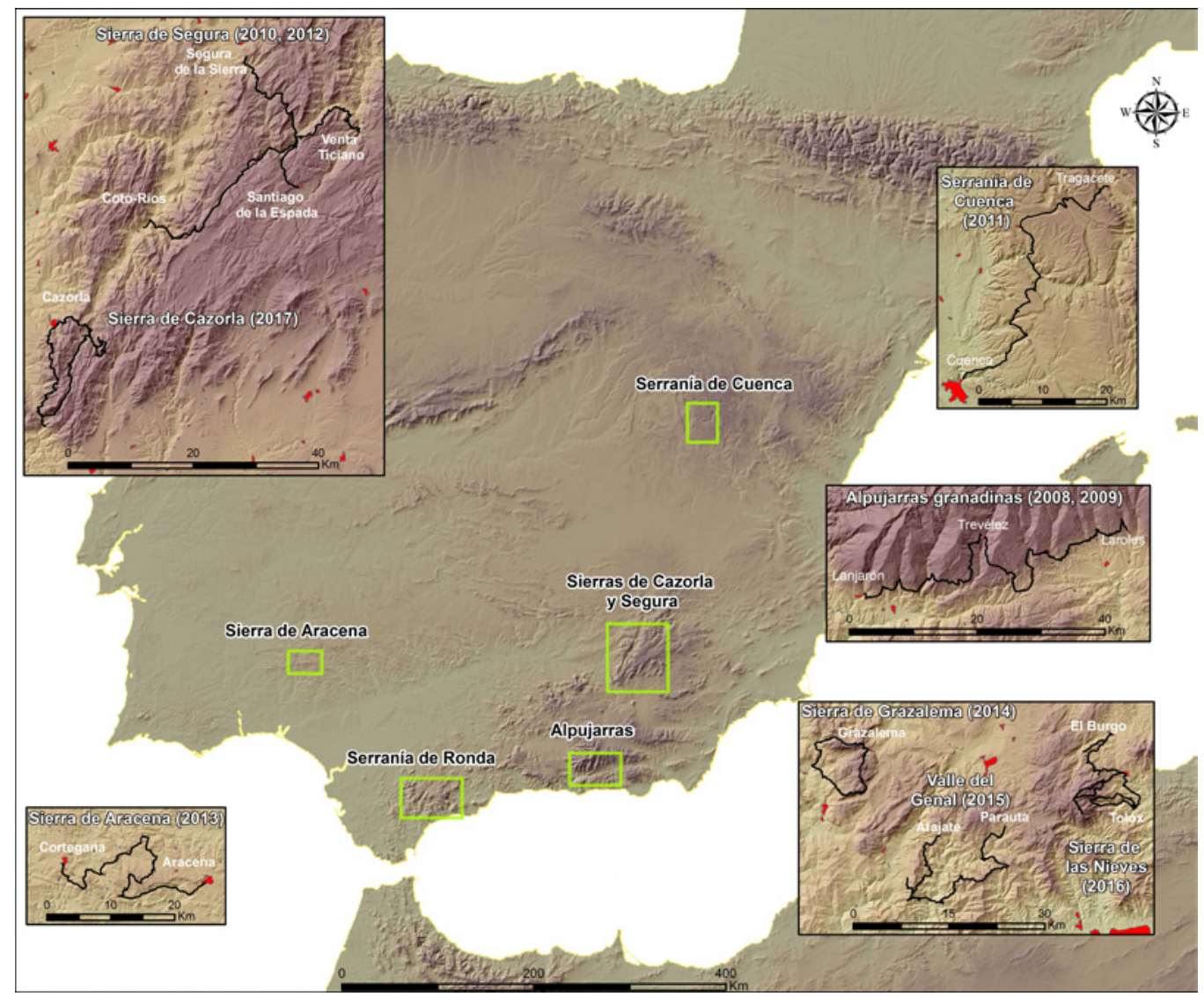

FIgURA $^{\mathrm{o}}$ 1: Localizaciones de las diez ediciones de la Salida Itinerante de Geografía 2008-2016. Elaboración propia

Aspectos relativos a la geomorfología, biogeografía, climatología, hidrología, geografía rural, ordenación del territorio, planeamiento urbanístico o paisaje son intencionadamente observados "in situ" y de forma conjunta.

b) Aprender (y enseñar) a desenvolverse en el medio natural utilizando instrumentos básicos del trabajo de campo en geografía, tanto clásicos (cartografía en papel, GPS, brújula), como las recientes aplicaciones que los teléfonos móviles incorporan (cartografía digital offline, realidad aumentada, etc): se trata de descubrir a los alumnos la actividad de caminar, no solo como un canal de disfrute y aprendizaje, sino como parte importante de la profesión del geógrafo.

c) Trabajar en equipo; el mero hecho de que un conjunto de personas conviva durante una serie de días, superando las dificultades propias de la ruta hasta alcanzar el 
destino fijado, suele suponer una satisfacción personal que revierte en una mayor integración del grupo. A ello habría que añadir que acostumbrarse a trabajar en equipo, al tiempo que intercambiando conocimientos con otros equipos, es uno de los objetivos principales de la pedagogia actual.

Para la consecución de estos objetivos, la salida se organiza a partir de unos contenidos curriculares (conceptuales, procedimentales y actitudinales) muy en consonancia con una parte importante de la estructura modular básica de los planes de estudios de Geografía. Estos contenidos se abordan a partir de diversas líneas temáticas (Tabla ${ }^{\circ}$ 2), donde adquieren especial relevancia las habilidades instrumentales y la capacidad de poner en práctica herramientas propias de los contenidos más aplicados de las materias (contenidos procedimentales); y las competencias basadas en valores y principios éticos (contenidos actitudinales).

Con respecto a esto último, uno de los principios inspiradores de esta iniciativa didáctica es la de trasladar al alumnado la premisa de la Geografía como palanca de transformación social. El carácter ético y comprometido de esta disciplina con los valores basados en la democracia, la justicia social o el respeto medioambiental (Martínez Fernández, 2014; Souto González, 2007; Zoido Naranjo, 2012) constituye el pilar fundamental de los contenidos actitudinales de la propuesta docente. Ello se trasmite al alumnado tanto en el desarrollo de sus materiales de trabajo como en el contacto personal y humano a lo largo de la experiencia docente.

\begin{tabular}{|l|l|l|}
\hline \multicolumn{1}{|c|}{$\begin{array}{c}\text { Líneas } \\
\text { temáticas }\end{array}$} & \multicolumn{1}{|c|}{ Contenidos } & \multicolumn{1}{c|}{ Procedimientos, competencias, roles } \\
\hline $\begin{array}{l}\text { Relieve y } \\
\text { suelos }\end{array}$ & $\begin{array}{l}\text { Contextualización espacial y temporal en } \\
\text { el marco geológico español (peninsular), } \\
\text { estudio de las particularidades geomorfo- } \\
\text { lógicas, litológicas y edáficas del ámbito } \\
\text { de estudio (materiales, formas y proce- } \\
\text { sos). }\end{array}$ & $\begin{array}{l}\text { - Búsqueda de datos y expresión gráfica } \\
\text { (p.e. elaboración/comentario de perfiles to- } \\
\text { pográficos y geológicos) } \\
\text { - Capacidad de abstracción y simplificación, } \\
\text { para explicar a los compañeros la compleji- } \\
\text { dad de los procesos geomorfológicos. }\end{array}$ \\
\hline $\begin{array}{l}\text { Clima y } \\
\text { aguas }\end{array}$ & $\begin{array}{l}\text { Contextualización general, estudio de los } \\
\text { elementos climáticos de la región (pre- } \\
\text { cipitación y temperatura) y búsqueda de } \\
\text { factores explicativos. } \\
\text { Caracterización longitudinal y transversal } \\
\text { de las principales cuencas hidrográficas } \\
\text { del ámbito, estudio del régimen fluvial y } \\
\text { su variabilidad interanual. }\end{array}$ & $\begin{array}{l}\text { - Búsqueda de datos climáticos y elabora- } \\
\text { ción grafos específicos (p.e. climogra- } \\
\text { mastán y diseño de gráficos al uso (perfiles } \\
\text { ración } \\
\text { longitudinal y transversal, régimen flu- } \\
\text { vial...) }\end{array}$ \\
\hline
\end{tabular}




\begin{tabular}{|c|c|c|}
\hline $\begin{array}{l}\text { Líneas } \\
\text { temáticas }\end{array}$ & Contenidos & Procedimientos, competencias, roles \\
\hline $\begin{array}{l}\text { Vegetación y } \\
\text { fauna }\end{array}$ & $\begin{array}{l}\text { Marco biogeográfico, factores y caracteri- } \\
\text { zación taxonómica y corológica, análisis } \\
\text { de las principales formaciones vegetales } \\
\text { naturales y antrópicas. } \\
\text { Caracterización e identificación de las } \\
\text { principales especies vegetales presentes } \\
\text { en la zona. } \\
\text { Elaboración de una pequeña guía de plan- } \\
\text { tas y fauna. }\end{array}$ & $\begin{array}{l}\text { - Búsqueda de datos específicos y elabora- } \\
\text { ción/representación de los mismos (mapas, } \\
\text { esquemas y transectos sobre la distribución } \\
\text { de las comunidades vegetales). } \\
\text { - Protagonismo constante, al ser conocedo- } \\
\text { res de las principales especies que encon- } \\
\text { tramos durante la travesía. Ese rol a veces } \\
\text { lo potencian con el planteamiento de juegos } \\
\text { sobre reconocimiento de especies. }\end{array}$ \\
\hline $\begin{array}{l}\text { Tecnologías } \\
\text { de la } \\
\text { Información } \\
\text { Geográfica } \\
\text { (TIG) }\end{array}$ & $\begin{array}{l}\text { Descripción pormenorizada de la ruta y } \\
\text { creación de las grafías esenciales del viaje } \\
\text { como el mapa general del recorrido, los } \\
\text { mapas de jornada y los perfiles topográfi- } \\
\text { cos de la itinerancia. }\end{array}$ & $\begin{array}{l}\text { - Manejo de los Sistemas de Información } \\
\text { Geográfica, y de datos asociados (MDE, } \\
\text { ortofotos). } \\
\text { - Rol de liderazgo, al conocer de primera } \\
\text { mano las especificidades y los principales } \\
\text { hitos de la ruta. Suelen encabezar el grupo } \\
\text { y son responsables del manejo y la carga de } \\
\text { los walki-talkies. }\end{array}$ \\
\hline $\begin{array}{l}\text { Desarrollo } \\
\text { territorial }\end{array}$ & $\begin{array}{l}\text { Búsqueda y sistematización de informa- } \\
\text { ción socioeconómica, que conduce a un } \\
\text { índice sintético de desarrollo territorial de } \\
\text { la comarca a partir de variables medioam- } \\
\text { bientales, de crecimiento económico y de } \\
\text { progreso social. }\end{array}$ & $\begin{array}{l}\text { - Aproximación a las principales fuentes de } \\
\text { información territorial, cálculo de estadísti- } \\
\text { cos e integración de variables. } \\
\text { - Familiarización con el trabajo de campo } \\
\text { en la esfera social (cuaderno, preguntas a } \\
\text { los habitantes...) }\end{array}$ \\
\hline $\begin{array}{l}\text { Ordenación } \\
\text { del territorio } \\
\text { y paisaje }\end{array}$ & $\begin{array}{l}\text { Análisis de los principales instrumentos } \\
\text { de planificación territorial del ámbito vi- } \\
\text { sitado. Aproximación a los enfoques ver- } \\
\text { tical y horizontal del análisis del paisaje } \\
\text { (mapas y fotografías). Búsqueda de refe- } \\
\text { rencias artísticas (literarias y pictóricas) } \\
\text { del ámbito visitado. }\end{array}$ & $\begin{array}{l}\text { - Familiarización con los principales instru- } \\
\text { mentos de planificación territorial y urbanística. } \\
\text { - Sensibilidad territorial y paisajística, } \\
\text { como conocedores de los principales valo- } \\
\text { res estéticos de ese territorio, así como de } \\
\text { los posibles conflictos e impactos antrópi- } \\
\text { cos. Moderadores de debates sobre transfor- } \\
\text { maciones del medio. }\end{array}$ \\
\hline $\begin{array}{l}\text { Historia y } \\
\text { antropología }\end{array}$ & $\begin{array}{l}\text { Descripción general de la evolución } \\
\text { histórica del ámbito de estudio y de sus } \\
\text { conexiones con la historia nacional o re- } \\
\text { gional. Desglose de las actividades tradi- } \\
\text { cionales de la comarca y puesta en valor } \\
\text { de la Administración. } \\
\text { Recopilación hemerográfica sobre hechos } \\
\text { recientes, detectando los temas que más } \\
\text { preocupan en la comarca durante los úl- } \\
\text { timos años. }\end{array}$ & $\begin{array}{l}\text { - Exploración de fuentes de información } \\
\text { complementaria menos convencionales y } \\
\text { a escala local: prensa comarcal, estudiosos } \\
\text { locales, etc. } \\
\text { - Familiarización con el trabajo de campo } \\
\text { (cuaderno, cuestionario dirigido a oriun- } \\
\text { dos...) }\end{array}$ \\
\hline
\end{tabular}

$\operatorname{TABLA~N}^{\circ} 2$ : Líneas temáticas. Elaboración propia 


\subsection{Desarrollo de la actividad}

Desde principios de curso y hasta la realización de la Salida, que tiene lugar a finales de abril, se lleva a cabo una serie de actividades relacionadas con la organización del evento (Figura $\mathrm{n}^{\circ} 2$ ). Los criterios principales a la hora de elegir la ruta son los siguientes: el interés del territorio a atravesar, la disponibilidad de lugares para pernoctar (los alojamiento no deben distar entre sí más de $20 \mathrm{~km}$ ), las características del sendero y su facilidad para recorrerlo (en condiciones de seguridad) y la disponibilidad presupuestaria.

Varias semanas antes, se realiza una salida previa por parte de un pequeño grupo de profesores y colaboradores (coincidiendo con el periodo no lectivo de Semana Santa) con la intención de conocer el entorno, trazar con precisión el recorrido, guardándolo mediante un track GPS, y seleccionar los puntos más adecuados para las exposiciones temáticas. Asimismo y durante este periodo, se realiza una convocatoria para que los posibles participantes sean informados de la ruta y se inscriban. En el caso de que los interesados superen el número máximo de participantes establecido (15), se lleva a cabo una selección basada en una serie de criterios académicos. Al alumnado seleccionado se le informa del equipamiento indispensable para la realización de la actividad ${ }^{6}$.

Una vez inscrito el alumnado participante, se forman diferentes grupos para cada una de las líneas de trabajo propuestas. Los contenidos de estas líneas varían en cada edición en función de las preferencias de los estudiantes, del perfil docente del profesorado y las particularidades de la zona. Cada grupo es responsable de elaborar un informe temático previo, que se incorpora al dossier general repartido el primer día de la travesía. Igualmente, cada estudiante debe preparar durante esta fase la exposición que realizará en el campo.

Así, dossier escrito - elaborado previamente a la salida pero modificado con posterioridad - y exposición oral — durante la salida - constituyen los dos principales outputs sobre los que los estudiantes deben trabajar. Dado que cada alumno se integra en un grupo con una temática específica, este se especializa activamente en una serie de contenidos particulares, pero recibe una enseñanza compartida de las otras temáticas por medio de sus compañeros.

Este dossier constituye un material proactivo de consulta a lo largo de la salida. Así, por poner un ejemplo, el inventario biogeográfico que elabora previamente el grupo de vegetación y fauna sirve para identificar las distintas especies que aparecen en el

6 Mochila mediana, botas de montaña, cantimplora, linterna, impermeable, mudas, gorra y crema solar. Adicionalmente se recomienda la instalación de aplicaciones útiles durante la itinerancia en sus dispositivos móviles: Oruxmap (uso de mapas offline para grabar o seguir rutas), Arbo1App y Aves de España (guía de árboles y avifauna), Cimas de España (realidad aumentada del relieve), Alpify (gestión de emergencias y primeros auxilios). 
recorrido. Cuando surge alguna duda circunstancial en la identificación o caracterización taxonómica de alguna planta, huella o excremento, se discute con el resto del grupo, teniendo como apoyo fundamental la información recogida en el dossier. Pero también hay que señalar que durante toda la actividad los estudiantes deben recopilar información y tomar contacto con actores locales con el fin de consolidar (y corregir, si fuese necesario) los contenidos recogidos en el dossier. Para ello, se acompañan de un cuaderno de campo donde vuelcan parte de esa información.

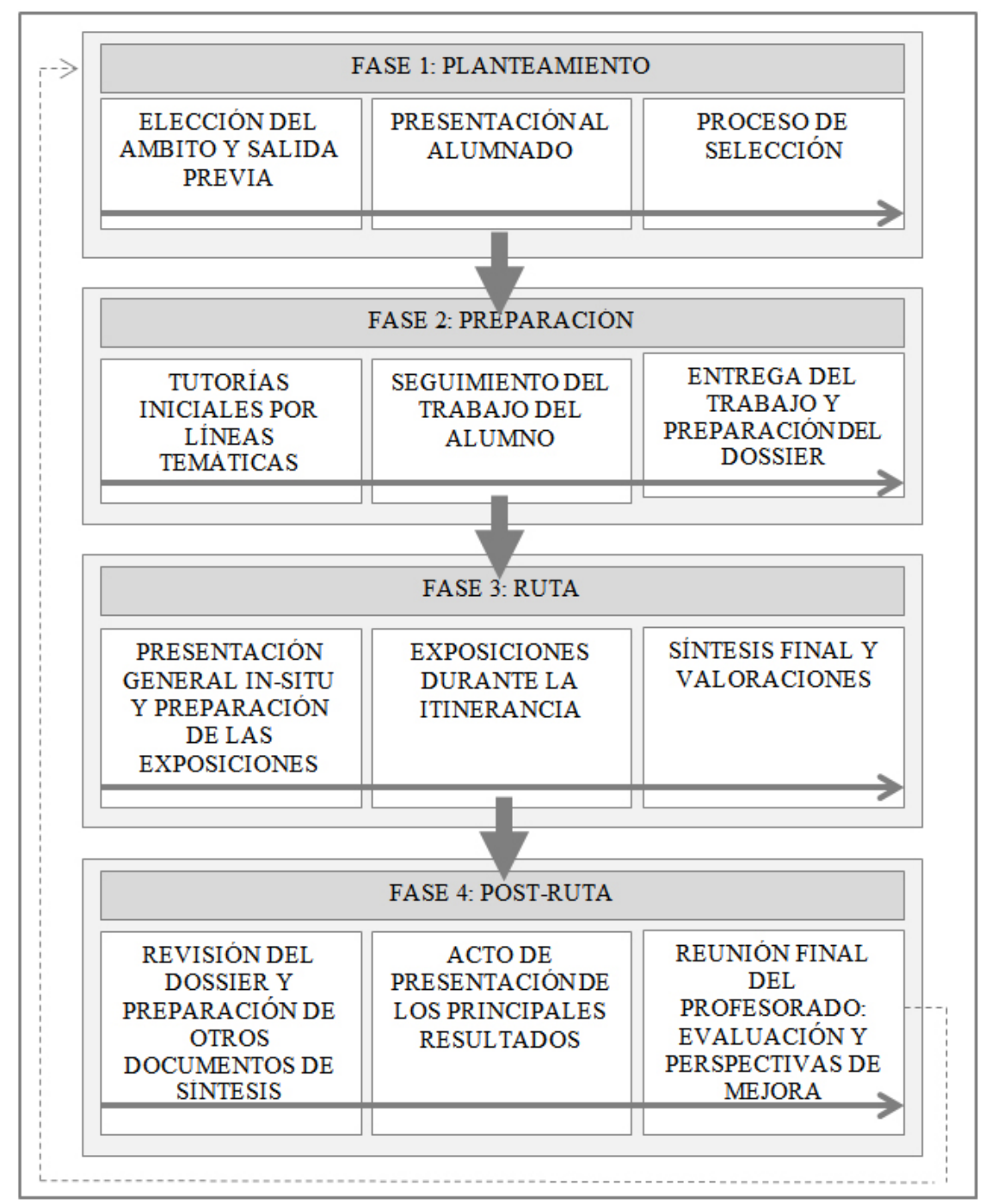

FIGURA N $^{0} 2$. Fases de la Salida Itinerante en Geografía. Elaboración propia 
La travesía tiene lugar en el periodo coincidente con la Feria de Abril de Sevilla, aprovechando la ausencia de clases lectivas de esta Universidad, y suele tener una duración de cuatro a cinco jornadas. El día de llegada al área de estudio se procede a planificar la secuencia de exposiciones de cada grupo.

Cada jornada se inicia con una explicación sobre el trazado, las dificultades, la localización de fuentes, manantiales y otros hitos, así como los puntos de parada para exposiciones y almuerzo (Figura $\mathrm{n}^{\mathrm{o}} 3$ ); por parte del grupo de TIG. El funcionamiento del grupo mientras marcha suele ser algo diferente a una caminata en fila india. Al frente se sitúan aquellos que ya conocen el itinerario, y dos alumnos se encargan, en los extremos del grupo, de estar en contacto mediante walki-talkies para mantener unido al conjunto y tratar de evitar posibles extravíos.

Aprovechando las menores temperaturas de la mañana, se emplean las primeras horas del día para realizar las subidas más fuertes y prolongadas, con la idea de alcanzar puntos de buena visibilidad antes del mediodía, y es en ellos donde tienen lugar las primeras exposiciones: el coordinador del grupo presenta tanto la zona como la materia, y los alumnos a los que corresponda exponen sus planteamientos. La parada larga en la que se realiza el almuerzo - con los alimentos que cada estudiante transporta individualmente - se lleva a cabo en zonas preferentemente sombrías y con vistas amplias. A menudo, y debido a la singularidad de los espacios que se eligen para almorzar, se suelen realizar exposiciones de líneas temáticas diversas. La segunda parte de la jornada suele extenderse hasta la puesta del sol, si bien en algunas ocasiones abarcó hasta entrada la noche. Favorecidos por la luz del atardecer, suele llevarse a cabo una segunda ronda de presentaciones ${ }^{7}$.

La duración de las jornadas no es uniforme, y depende de la distancia a recorrer: se procura la alternancia entre jornadas exigentes con otras más asequibles. Tras finalizar la última, una cena previamente concertada pone punto final a la travesía ${ }^{8}$, y

\footnotetext{
7 Alcanzado el lugar de pernoctación, que suelen ser una o varias casas rurales situadas en algún pequeño pueblo, llega uno de los momentos más críticos del día para el profesorado: la necesaria organización y reparto de casas y habitaciones, situación normalmente complicada por la falta de información precisa acerca de su cantidad y distribución, un insuficiente número de camas individuales, la preferencia de cada individuo por compartir el espacio con determinadas compañías, y la necesidad generalizada de aseo y comida.

${ }^{8}$ La organización del resto de cenas, dado que, por la estructura de las jornadas, representa la comida más importante, requiere de un lugar común y una implicación general: que sea costeada por todos y que, de una u otra manera, todos participen en su elaboración. Si hay tiempo disponible el coordinador del grupo se reúne con el alumnado para ultimar detalles de futuras exposiciones.
} 


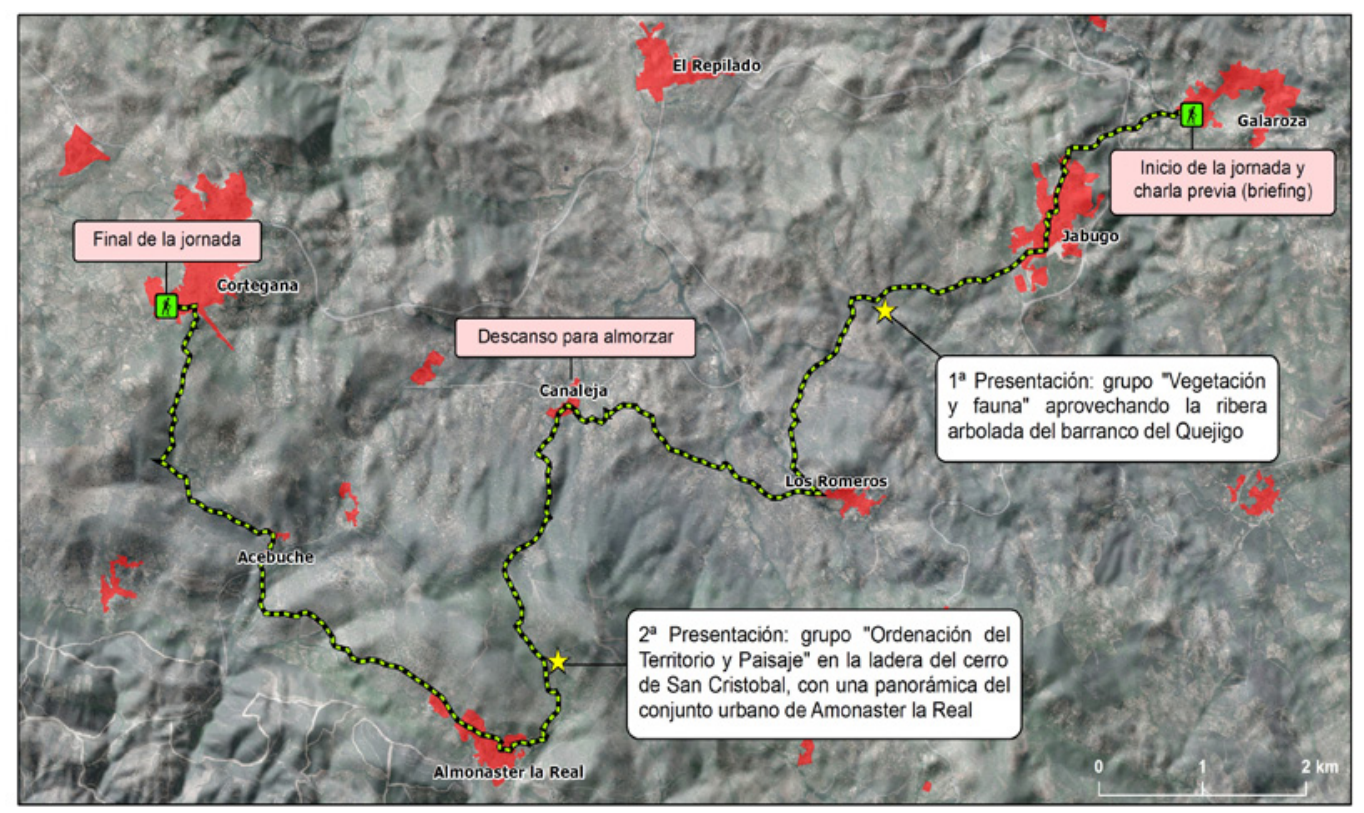

Jornada 4: Galaroza - Cortegana $(21,3 \mathrm{~km})$

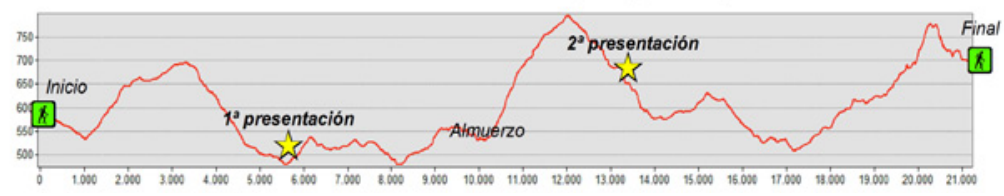

FigURA N ${ }^{\circ}$ 3: Esquema de una jornada (Galaroza-Cortegana, 2013).

Elaboración propia

ya en el viaje de vuelta, se realiza una puesta en común que sirve como síntesis final de la experiencia y valoración de sus resultados.

En las semanas posteriores a la travesía es común encontrar dificultades a la hora de revisar y sintetizar los trabajos realizados en el campo (al tratarse de fechas coincidentes con la preparación de los exámenes de junio). No obstante, finalmente se materializan los principales documentos de síntesis: una versión revisada del dossier, un póster sintético (cartografía, perfil altimétrico, fotografía...) y un cortometraje que resume la experiencia. El alumnado, especialmente aquellos aficionados a la fotografía, suben las mejores instantáneas a Geophotopedia, un repositorio fotográfico gratuito y on-line de imágenes con contenido territorial que cuenta ya con unos 250 colaboradores y más de 12.000 imágenes (Ver Fraile Jurado et al, 2016). Finalmente, se lleva a cabo un acto de presentación de los resultados y una reunión del profesorado que evalúa y establece perspectivas de mejora. 


\section{EVALUACIÓN DE LA ACTIVIDAD}

Con el propósito valorar los efectos de la Salida Itinerante entre el alumnado durante los últimos diez años, se ha realizado una encuesta dirigida a los participantes. Se ha llevado a cabo en dos fases, durante el verano de 2016 y durante el invierno de 2017. Consiste en siete preguntas cerradas y dos abiertas, que se encuentran recogidas en la figura $n^{\circ} 4$.

Los resultados obtenidos indican un alto grado de satisfacción por parte del alumnado que ha participado en alguna de las sucesivas ediciones de la Salida Itinerante. Existe un consenso casi absoluto en cuanto a las cuestiones referentes a recomendar la participación en la actividad a otros compañeros de estudios o sobre la afirmación de que la Salida cambió su percepción de los estudios de Geografía. Más de dos tercios de los encuestados indicaron haber adquirido nuevos conocimientos a los que en el aula no suelen tener acceso. Se apreció un tono levemente más crítico en la valoración de los métodos empleados para el aprendizaje, puesto que si bien el 90\% de la evaluación fue mayoritariamente positiva, el grueso de estas respuestas se centraron en la valoración de "Bastante de acuerdo" frente a una minoría de "Muy de acuerdo".

Asimismo, más de dos tercios de los participantes consideraron que la proporción de contenidos entre Geografía Física y Geografía Humana fue adecuada, mientras que la cantidad de estudiantes que la consideraron inadecuada fue semejante con respecto a ambas disciplinas. Se obtuvieron resultados parecidos en cuanto a la proporción de contenidos académicos y relaciones sociales. En relación a las cuestiones con respuesta abierta, los estudiantes apenas recuerdan aspectos negativos, evidenciándose un tono positivo generalizado.

Las cuestiones de respuesta abierta han permitido profundizar algo más en la valoración del alumnado. En ese sentido destacamos los siguientes aspectos: el fomento de las habilidades de comunicación oral ("me aportó una serie de habilidades en cuanto a la sintetización, esquematización y presentación de datos, así como para ejercitar la comunicación en público”); el origen o el fortalecimiento de relaciones personales ("amplié el círculo de colegas gracias al buen ambiente de esta convivencia académica"); la satisfacción por la actividad deportiva ("aporta experiencia en cuanto al senderismo y su logística"); y el enriquecimiento general provocado por la alteración de los vectores tradicionales de enseñanza y aprendizaje ("pude incrementar mis conocimientos gracias al aporte de los compañeros no solo en las exposiciones sino durante todo el recorrido"). 


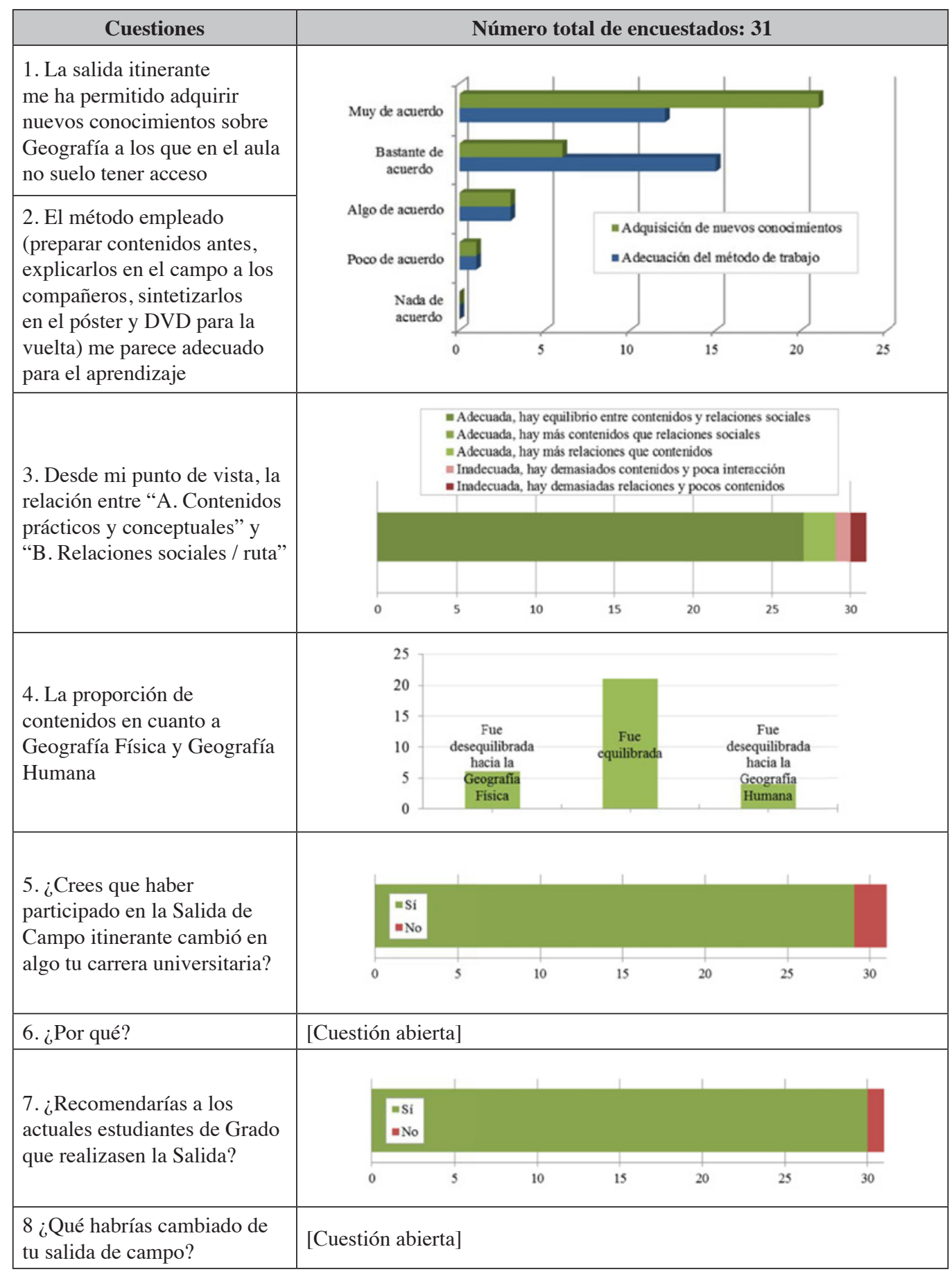

Figura No 4 : Síntesis de la evaluación del alumnado 


\section{VALORACIONES FINALES}

A modo de conclusión, se presentan a continuación los aspectos más relevantes en relación con el trabajo desarrollado: la convivencia académica, las metodologías docentes, la vinculación con la actividad deportiva, la idoneidad de ciertos territorios, el proceso de retroalimentación y la integración curricular de la actividad.

\subsection{La convivencia académica}

La Salida Itinerante es también una convivencia académica al aire libre y pone en contacto a los alumnos del Grado de Geografía, inaugurando o estrechando vínculos entre personas de similar o distinta edad, curso y/o motivación (Figura $n^{\circ} 5$ ). Se observa que el aprendizaje va más allá de las exposiciones y que durante el camino se producen charlas muy interesantes entre profesores y alumnos, y entre los propios estudiantes en un aprendizaje entre iguales. En este sentido se puede destacar el rol desempeñado por los alumnos séniores que, en algunas ocasiones, compatibilizan sus estudios de geografía con actividades profesionales cercanas al territorio (geólogo, antropólogo, historiador, arquitecto, bombero forestal...); estos alumnos transmiten sus conocimientos al resto del colectivo, incluyendo los profesores. En segundo lugar ha de subrayarse el papel de los alumnos de los cursos más avanzados, que ya han escogido un perfil de formación e investigación y que, por tanto, también se convierten en vehículos de aprendizaje hacia los estudiantes menos experimentados. En este particular, destaca la visión de Álvarez, Vázquez y Rodríguez (2016), que analizan y subrayan, en una investigación reciente, la influencia de esta actividad en la formación inicial del profesorado universitario (estudiante de apoyo, becario en formación...). Finalmente, cabe destacar la participación, en ocasiones, de estudiantes oriundos del lugar, aspecto muy útil desde el punto de vista logístico pero también muy enriquecedor en lo pedagógico.

El rol del profesorado en esta convivencia académica también es una cuestión interesante. Para sacar el máximo provecho a esta actividad nos preguntamos si deben mantenerse las distancias del aula o, en cambio, integrarnos más en el grupo, minimizándose el estatus de profesor. Este reto se agudiza si consideramos que el perfil no es homogéneo, encontrando desde docentes noveles a profesores veteranos.

La Salida Itinerante es una experiencia global donde se busca que haya una buena relación entre todos los integrantes, que permita el goce del entorno natural, y que se obtengan unos resultados académicos. Estos objetivos deben ser cuidadosamente jerarquizados, ya que en determinados momentos pueden entrar en conflicto. Con la experiencia vivida estimamos como más importante el mantenimiento de unas buenas relaciones entre los alumnos y profesores; esto hace que se creen más y mejores lazos 
entre todos y que aumente la confianza, punto de partida muy útil para la sensación de seguridad y el buen ambiente a lo largo del recorrido. En este marco, el mejor vehículo para preservar la auctoritas durante la itinerancia es una buena tutorización previa; es aquí donde el profesor sí se centra en los resultados académicos, asegurándose que los alumnos que tutela asimilen los principales contenidos y realicen una motivante exposición al resto de compañeros.

\subsection{Las metodologías docentes}

La principal carencia de la Salida Itinerante - que deriva en parte de su falta de integración curricular - es la dificultad real para completar un ciclo completo de aprendizaje aula-campo- aula. Siguiendo a Llancavil (2015), que sugiere una "metodología indagatoria", la actividad quedaría dividida en las siguientes fases: focalización (relato del ámbito y preguntas iniciales), exploración (búsqueda de fuentes, salida y entrevistas in situ), reflexión (síntesis, organización y análisis), aplicación (transferencia del conocimiento a otros ámbitos y problemáticas) y evaluación (metacognición del proceso). Las dos últimas fases comprenden las 3 preguntas clave que debe hacerse el alumnado después de la salida: ¿qué aprendí?, ¿cómo lo aprendí?, ¿para qué lo aprendí?. Entendemos que estas etapas deben reforzarse, siendo éste uno de los principales retos en el desarrollo futuro de esta experiencia.

Como una segunda perspectiva de mejora, entendemos que debe fortalecerse el rol del alumnado como máximo responsable del proceso de enseñanza-aprendizaje, especialmente en lo tocante a la exposición final de los resultados. Para ello la etapa de preparación se subdivide en dos fases: en la primera, los estudiantes recopilan información, sintetizan y elaboran un documento final que debe incluirse en el dossier general; en la segunda (a mejorar), cada equipo piensa en una estrategia y un guion para la exposición preparando, en cada caso, los materiales de la presentación (póster, cuestionario, juego de roles, puntos del debate...).

Finalmente, el fomento del cuaderno de campo es un tercer aspecto clave para fortalecer esta experiencia didáctica. Esta herramienta resulta clave para plasmar observaciones, nuevos conocimientos y consecuentes preguntas, pero también es el lienzo ideal para ilustrar la experiencia personal y, por qué no, las emociones del momento (Figura $\mathrm{n}^{\circ}$ 6). Si el alumnado encuentra dificultades al respecto, podrían plantearse soluciones intermedias mediante un proceso semi-guiado. Como referencias directas podemos destacar las aportaciones de Parra, Domínguez y Caballero (2008) o el reciente trabajo realizado por Cuello Gijón y Cuello Pérez (2016). Éste último se trata de una serie de Guías Didácticas estructuradas en 3 ejes - Fundamentos naturales, Procesos históricos y Percepción y representaciones - que, a partir de la realización de 
fichas independientes, van conduciendo a una lectura integral del paisaje. La creciente participación del alumnado del Doble Grado en Geografía e Historia hace especialmente interesante esta metodología, en la que el paisaje se convierte en el hilo conductor donde convergen geografía, historia y manifestaciones artísticas.
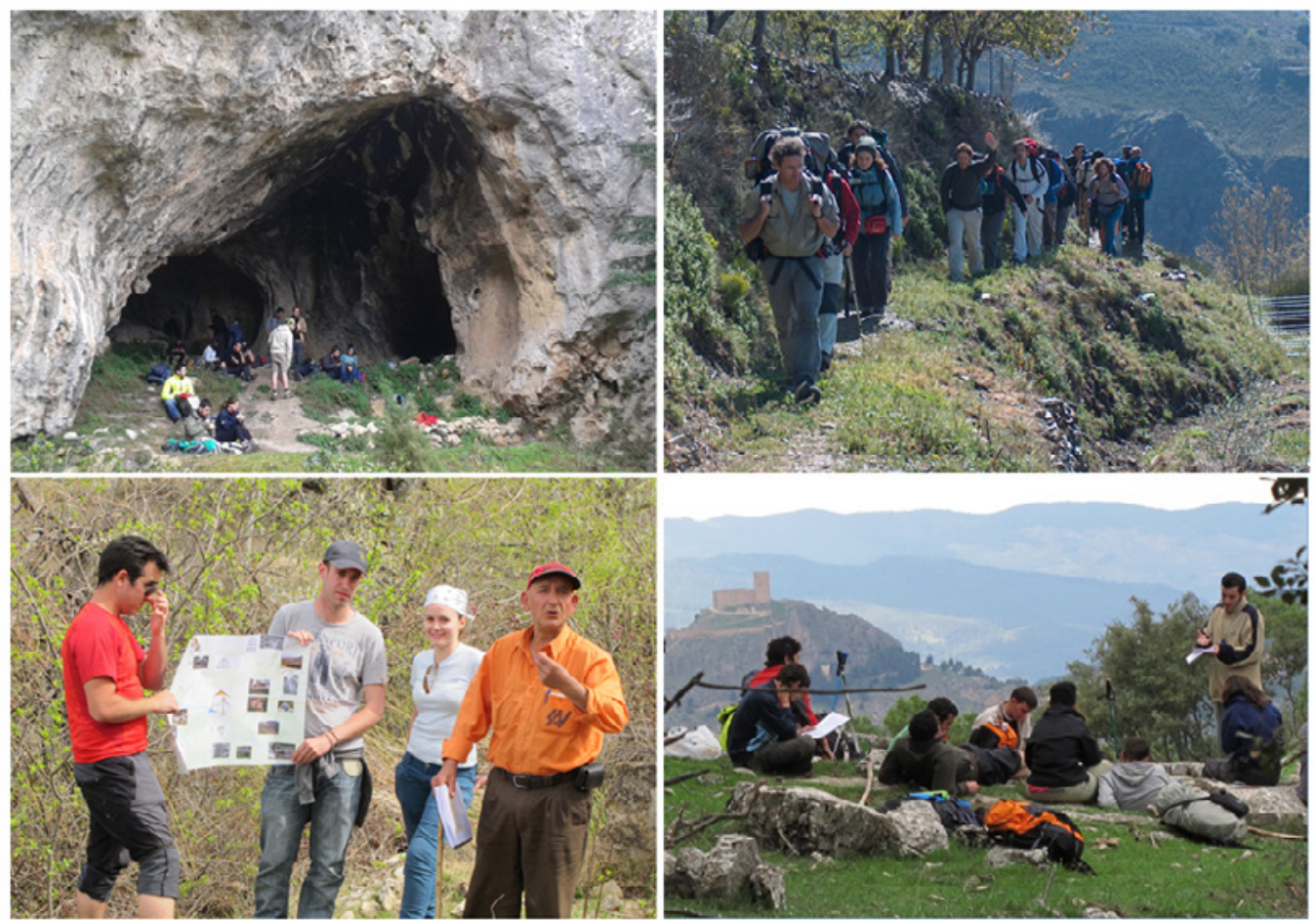

FIGURA $N^{\circ}$ 5. La convivencia académica

\subsection{El vínculo de la geografía con la salud y la actividad deportiva}

En tanto que el proyecto educativo es transversal, el ejercico físico y deportivo representa una parte importante de esta experiencia. Las largas caminatas, los senderos remotos y los acusados desniveles ponen a prueba la resistencia de los participantes. Y es que el acercamiento a los fenómenos que tienen lugar en el espacio geográfico implica una intensa interacción física y corporal entre el sujeto y el entorno natural, donde también median ciertas dosis de aventura que aportan una motivación añadida. Además, en esta salida itinerante los alumnos desarrollan unas habilidades específicas para desenvolverse en la montaña: lectura de mapas, manejo de dispositivos GPS, preparación de una mochila con enseres básicos, uso del cuaderno de campo, etc. 


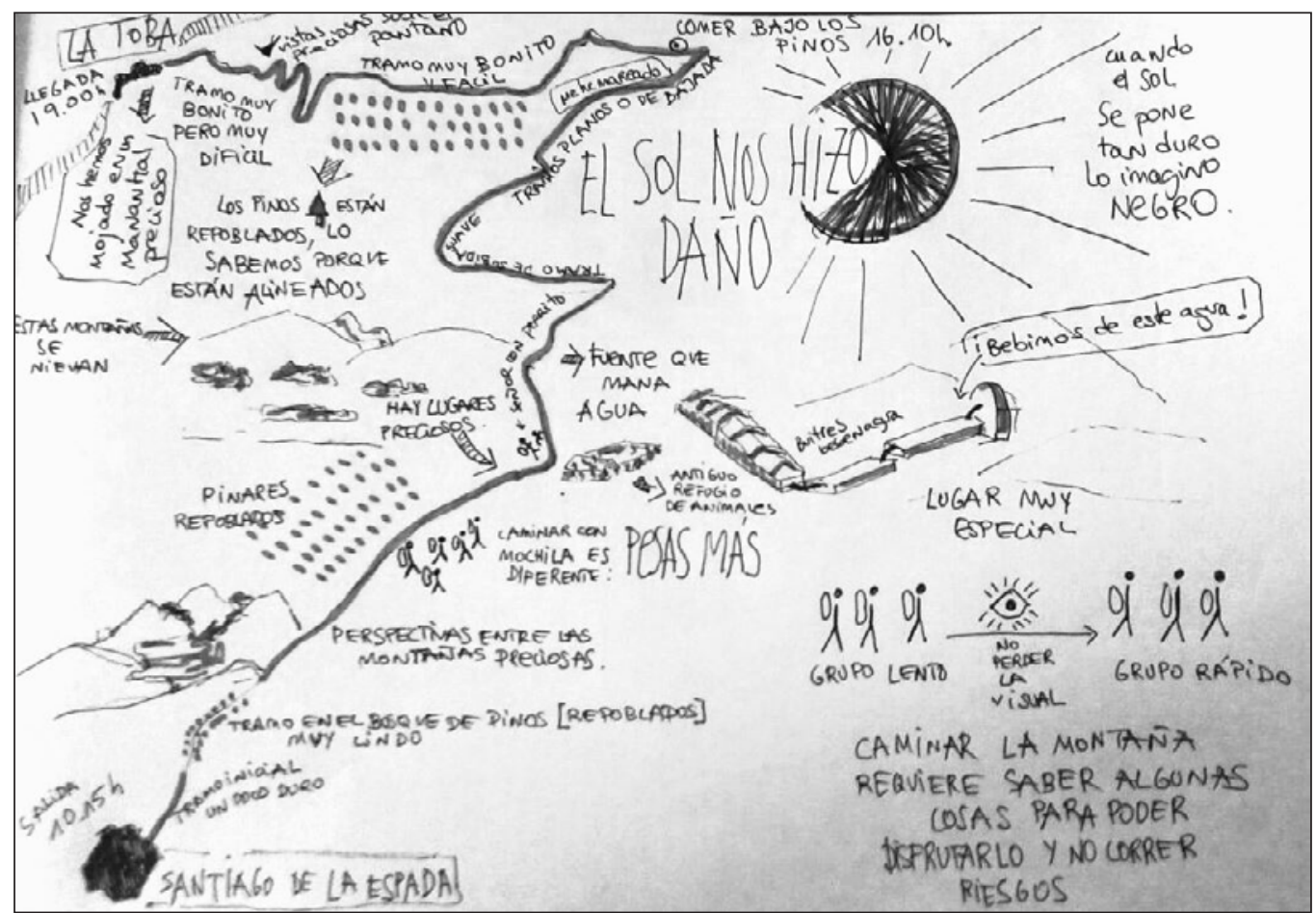

FiguRA N ${ }^{\circ}$ 6. El cuaderno de campo. María Isabel Martín Ruiz

\subsection{La idoneidad de ciertos territorios "más transversales"}

Las sucesivas ediciones de la salida itinerante por diversos destinos han puesto de manifiesto que determinados territorios responden mejor ante el enfoque transversal de esta actividad. Sin menospreciar la riqueza y complejidad de todos los espacios recorridos, aquellos espacios serranos de vocación natural pero con un largo proceso de ocupación humana han resultado ser más versátiles para el estudio de sus aspectos físico-ambientales, económicos y socio-culturales. Esto se ha podido percibir en lugares como las Alpujarras, la sierra de Huelva o el valle del Genal, territorios de una intensa humanización donde las actividades agrícolas tienen un peso significativo en la conformación del paisaje rural resultante. También el modelo de poblamiento disperso, casi atomizado, pero regularmente distribuido por todo el territorio en estas comarcas favorece una organización más flexible de las jornadas, al poder contar con una oferta de alojamientos espacialmente diversificada. Por otra parte, algunos de estos espacios están amparados por figuras de protección (Parque Natural), un marco jurídico específico que también contribuye al estudio transversal de otras temáticas geográficas, como la planificación territorial y ambiental. 


\subsection{El proceso de retroalimentación flexible}

La actividad se somete a un proceso de auto-evaluación todos los años mediante la valoración in situ al final de la itinerancia y una reunión conclusiva del profesorado. Ello permite incidir en las perspectivas de mejora para la edición siguiente en relación con el trabajo académico, las características del recorrido, la convivencia y la organización. Resultado de este ejercicio de feedback, se han implementado cambios a propuesta de los propios alumnos como, por ejemplo, el incremento de los días en ruta o la inclusión, en la medida de lo posible, de alguna jornada circular (donde se sale y se llega al mismo destino, permitiendo caminar con una mochila de menor peso). Igualmente, la flexibilidad de la actividad incluye la incorporación de elementos nuevos para la itinerancia y las exposiciones, en consonancia con el avance en las tecnologías (tabletas, aplicaciones móviles de geolocalización, vegetación y fauna, primeros auxilios...). Por otra parte, el presente artículo - en la medida que presenta los principales resultados de una auto-evaluación general más extensa - nos ha ayudado a realizar un balance de toda la trayectoria de esta actividad académica y, en consecuencia, a proponer nuevas perspectivas a corto y largo plazo.

\subsection{Hacia la integración curricular}

El carácter voluntario de esta propuesta extracurricular conlleva la dificultad de reunir a un grupo de 15 estudiantes y 5 profesores para que lleven a cabo una actividad sacrificando su tiempo libre durante el periodo vacacional (Feria de Abril). Pero también representa una ventaja al considerar que, aun así, si algunas personas deciden participar es porque muestran una motivación adicional, aspecto que favorece mucho el proceso de enseñanza-aprendizaje y la convivencia académica y social.

Con todo, esta actividad podría consolidarse como una propuesta reglada en los currículos educativos de la geografía universitaria, ya sea en la forma de una asignatura en los últimos cursos de la titulación o con alguna otra fórmula docente. Así lo vienen haciendo escuelas de gran tradición como la anglosajona - tanto en Reino Unido (McGuinness y Simm, 2005) como en EE.UU. (Mullens, Bristow y Cuper, 2012)-, en cuyos grados de Geografía ya existe una salida de campo de varios días (incluso de ámbito internacional), lo que supone una culminación del proyecto docente en términos similares a lo que representa el Trabajo Fin de Grado.

\section{AGRADECIMIENTOS}

Agradecemos a los departamentos de Geografía de la Universidad de Sevilla su colaboración y financiación, y en particular a aquellos de sus miembros que han cooperado directamente. Como no podía ser menos, también damos las gracias a todos los alumnos participantes. 


\section{REFERENCIAS}

Almuedo Palma, J., 2016, "Los paseos escolares en las escuelas sevillanas a principios del xx: algo más que un intento de innovación didáctica" en L. Alanís et al, eds. Nativos digitales y geografía en el siglo XXI: Educación geográfica y sistemas de aprendizaje. Sevilla: Universidad Pablo de Olavide y Grupo de Didáctica de la AGE, pp. 436-440.

Álvarez Piñeros, D.; Vázquez Ortiz, W.F. y Rodríguez Pizzinato, L.A., 2016. „La salida de campo, una posibilidad en la formación inicial docente“. Didáctica de las Ciencias Sociales y Experimentales, 31, pp. 61-77.

Barraqué Nicolau, G., 1978.Metodología de la Enseñanza de la Geografía. La Habana: Libros para la Educación.

Careri, F., 2002.Walkscapes: el andar como práctica estética/walking as anaestheticpractice. Barcelona: Gustavo Gili.

Crespo Castellanos, J.M., 2012. "Un itinerario didáctico para la interpretación de los elementos físicos de los paisajes de la Sierra de Guadarrama". Didáctica Geográfica, 13, pp. 15-34.

Cuello Gijón, A. y Cuello Pérez, M.I., 2016.La carretera enseña sus paisajes. Guías didácticas. Sevilla: Junta de Andalucía, Centro de Estudios Paisaje y Territorio y Universidad de Sevilla.

Delgado Bujalance, B. y Ojeda Rivera, J., 2007. "El viaje pedagógico como método de conocimiento de paisajes. Aplicación a Andalucía”. Investigaciones Geográficas, 44, pp. 5-31.

Delgado Bujalance, B.; Torres Gutiérrez, F.J.; García García, A. y Ojeda Rivera, J.F., 2013. "El viaje como aprendizaje significativo y pertinente. Crónica de una experiencia andaluza" en P. Paneque y J.F. Ojeda, eds. El viaje en la geografía moderna. Sevilla: Universidad Internacional de Andalucía, pp. 429-444.

Fraile Jurado, P., Sánchez Escalera, D. y Medina Vizuete, B., 2016. "El uso de la fotografía como recurso docente para la interpretación del territorio en el aula: las potencialidades de geophotopedia”. Didáctica Geográfica, 17, pp. 63-78.

Fuller, I.; Edmondson, S.; France, D.; Higgitt, D. y Ratinen, I., 2006. "International Perspectives on the Effectiveness of Geography Fieldwork for Learning".Journal of Geography in Higher Education, 30(1), pp. 89-101.

García de la Vega, A., 2004. "El itinerario geográfico como recurso didáctico para la valoración del paisaje". Didáctica Geográfica, 6, pp. 79-95.

García Farrero, J., 2014. "Presencia de la pedagogía en el acto de caminar: homoviātor, nomadismo y formación". Teoría de la Educación; Educación y Cultura en la Sociedad de la Información, 15(4), pp. 56-84. 
García Martín, M., 2014. "Conflictos territoriales en torno al agua: salir al campo como metodología de enseñanza" en Martínez Medina, R. y Tonda Monllor, E.M., eds. Nuevas perspectivas conceptuales y metodológicas para la educación geográfica. Córdoba: Grupo de Didáctica de la Geografía de la AGE- Universidad de Córdoba, vol. 2, pp. 179-194.

Godoy, I. y Sánchez, A., 2007.’El trabajo de campo en la enseñanza de la Geografía”. Sapiens, 8 (2), pp. 137-146.

Gómez Mendoza, J. y Sanz Herráiz, C., 2013. "La excursión geográfica universitaria con fines formativos e investigadores: su consolidación en la Escuela Española” en P. Paneque y J.F. Ojeda, eds. El viaje en la geografía moderna. Sevilla: UNIA, pp. 289-328.

Gros, F., 2014. Andar, una filosofía. Madrid: Taurus.

Lara de González, S., 2011. "El trabajo de campo desde la perspectiva del docente". Sapiens, 12(1), pp. 76-93.

Llancavil Llancavil, D., 2015. "Uso de metodología indagatoria para la enseñanza del espacio geográfico”. Giramundo, 2(3), pp. 39-49.

Márquez Pérez, J. et al. 2016. "La salida itinerante de geografía”. XI Congreso nacional Didáctica de la geografía. Universidad Pablo de Olavide (Sevilla). 18-19 de Noviembre de 2016.

Martínez Fernández, L. C. 2014. "Educación para la paz y la igualdad: una propuesta de contenidos desde la Geografía”. Tabanque Revista pedagógica, 27, pp. 217-234.

McGuinness, M., y Simm, D., 2005. “Going global? Long-haul fieldwork in undergraduate geography”. Journal of Geography in Higher Education, 29(2), pp. 241-253.

Mullens, J. B.; Bristow, R. S. y Cuper, P., 2012. "Examining trends in international study: A survey of faculty-led field courses within American departments of geography". Journal of Geography in Higher Education, 36(2), pp. 223-237.

Opezzo, M. y Schwartz, D., 2014. "Give your ideas some legs: The positive effect of walking on creative thinking". Journal of Experimental Psychology: Learning, Memory and Cognition, 40(4), pp. 1142-1152.

Parra Bollero, M., Domínguez Carrillo, G. \& Caballero Blanco, P.J. 2008. "El cuaderno de campo. Un recurso para dinamizar senderos desde la educación en valores". Agora para la educación física y el deporte , 7-8, pp. 145-158.

Pérez de Sánchez, A.G. y Rodríguez Pizzinato, L.A., 2006. "La salida de campo: una manera de enseñar y aprender Geografía”. Geoenseñanza, 11(2), pp. 229-234.

Solnit, R., 2015. Wanderlust: una historia del caminar. Madrid: Capitán Swing. 
Sousa Fernandes, S.A.; García Monteagudo, D.\&Souto González, X.M, 2016. "Educación geográfica y las salidas de campo como estrategia didáctica: un estudio comparativo desde el Geoforo Iberoamericano". Biblio3W Revista Bibliográfica de Geografía y Ciencias Sociales, [En línea. Acceso libre]. Barcelona: Universidad de Barcelona, XXI, 1155, 5 de abril de 2016.<http://www.ub.es/geocrit/b3w-1155. pdf> [Último acceso 9 septiembre 2016]

Souto González, J. M.2007. “Educación geográfica y ciudadanía”. Didáctica Geográfica, 9, pp. 11-32.

Zoido Naranjo, F. 2012. "El paisaje un concepto útil para relacionar estética, ética y política". Scripta Nova, XVI, 407. 
